\title{
Uncovering the pathophysiology of irritable bowel syndrome by exploring the gut-brain axis: a narrative review
}

\author{
He-Yong Tang ${ }^{1 \#}$, Ai-Juan Jiang ${ }^{2 \#}$, Xi-Yang Wang ${ }^{1}$, Hao Wang ${ }^{2}$, Yuan-Yuan Guan ${ }^{3}$, Fei Li ${ }^{4}$, Guo-Ming Shen ${ }^{2}$ \\ ${ }^{1}$ Graduate School of Anhui University of Chinese Medicine, Hefei, China; ${ }^{2}$ School of Integrated Traditional Chinese and Western Medicine, Anhui \\ University of Traditional Chinese Medicine, Hefei, China; ${ }^{3}$ Department of Acupuncture and Moxibustion, The First Affiliated Hospital of Anhui \\ University of Traditional Chinese Medicine, Hefei, China; ${ }^{4}$ Department of Rehabilitation, Second Affiliated Hospital of Anhui University of Chinese \\ Medicine, Hefei, China \\ Contributions: (I) Conception and design: HY Tang, AJ Jiang; (II) Administrative support: GM Shen; (III) Provision of study materials or patients: H \\ Wang, YY Guan; (IV) Collection and assembly of data: H Wang, YY Guan, XY Wang; (V) Data analysis and interpretation: XY Wang, F Li, GM \\ Shen; (VI) Manuscript writing: All authors; (VII) Final approval of manuscript: All authors. \\ "These authors contributed equally to this work. \\ Correspondence to: Dr. Guo-Ming Shen. School of Integrated Traditional Chinese and Western Medicine, Anhui University of Traditional Chinese \\ Medicine, Hefei 230038, China. Email: shengm_66@163.com; Dr. Fei Li. Department of Rehabilitation, Second Affiliated Hospital of Anhui \\ University of Chinese Medicine, No. 300, Shouchun Road, Hefei 230061, China. Email: leagcen@21cn.com.
}

Objective: To improve the pathophysiological understanding of irritable bowel syndrome (IBS) by exploring the gut-brain axis.

Background: Disorders of gut-brain interaction (DGBIs) are gastrointestinal (GI) disorders in which alterations in bowel functions occur. IBS, which is one of the most studied DGBIs, is linked with abdominal distress or pain without obvious structural or biochemical anomalies.

Methods: The etiology of IBS has not been clearly described but is known to be multifactorial, involving GI motility changes, post-infectious reactivity, visceral hypersensitivity, gut-brain interactions, microbiota dysbiosis, small intestinal bacterial overgrowth, food sensitivity, carbohydrate malabsorption, and intestinal inflammation.

Conclusions: One of the main features of IBS is the occurrence of structural and functional disruptions in the gut-brain axis, which alter reflective and perceptual nervous system reactions. Herein, we provide a brief summary of this topic. Furthermore, we discuss animal models, which are important in the study of IBS, especially as it is linked with stressors. These animal models cannot fully represent the human disease but serve as important tools for understanding this complicated disorder. In the future, technologies, such as organ-on-a-chip models and metabolomics, will provide novel information regarding the pathophysiology of IBS, which will play an important role in treatment development. Finally, we take a brief glance at how acupuncture treatments may hold potential for patients with IBS.

Keywords: Pathophysiology; irritable bowel syndrome (IBS); gut-grain interaction; animal models; gastrointestinal disorders (GI disorders)

Submitted May 17, 2021. Accepted for publication Jul 14, 2021.

doi: 10.21037/atm-21-2779

View this article at: https://dx.doi.org/10.21037/atm-21-2779

\section{Introduction}

Disorders of gut-brain interaction (DGBIs) are the most common gastrointestinal (GI) disorders. Among these disorders, irritable bowel syndrome (IBS) is one of the most researched. IBS manifests as changes in bowel functions accompanied by abdominal discomfort or pain with a lack of demonstrable biochemical and structural anomalies (1). The etiology of IBS has yet to be fully described, but it 
is known to involve a variety of factors. Uncovering the pathogenesis of IBS is imperative for the development of pharmacotherapeutic agents (2). The pathogenesis of IBS entails GI motility alterations, post-infectious reactivity, visceral hypersensitivity, gut-brain interactions, microbiota dysbiosis, small intestinal bacterial overgrowth, sensitivity to food, carbohydrate malabsorption, and intestinal inflammation (2). There is not a definitive treatment for IBS. It is typically controlled by removing factors that exacerbate it, such as certain drugs, stressors, and dietary habits. However, modulating the gut-brain axis is being investigates as it an attractive target for the development of novel treatments (3).

Patients with IBS can typically be divided into those who predominantly have diarrhea and those who predominantly have constipation. However, some patients with the condition experience a combination of the two, and some patients might even switch from one bowel pattern to the other (4). The existence of highly variable bowel symptoms reinforces a diagnosis of IBS, but it is the co-occurrence of abdominal pain and agitated excretion that serves as a prerequisite for diagnosing the condition (5). The Rome IV criteria is used to classify IBS into four subtypes: IBS with diarrhea (IBS-D), IBS with constipation (IBS-C), IBS with a mixture of constipation and diarrhea symptoms (IBS-M), and IBS unclassified (IBS-U). The categorization of patients into these subtypes is based on the proportion of time experiencing hard or lumpy stools versus loose or watery stools reported by the patient (6). Traditional IBS management has been symptom based. However, due to its complex, multidimensional nature, treating the IBS subtypes with one agent successfully is not likely (3).

Population-based studies have revealed that IBS is extremely common $(5,7,8)$. For instance, a recent global study focusing on 24 countries utilized the Rome IV diagnostic questionnaire, Rome III IBS questions, and 80 items to identify variables associated with DGBIs. The results revealed that $40 \%$ of the global population suffers DGBIs which affect individuals' quality of life and health care use (9). In Western countries, the IBS population is typically predominated by females (10); however, this is not the case in the East (11). In Asia, IBS is believed to be under diagnosed (12).

This review provides a summary of the pathophysiology of IBS, with an emphasis on microbiota-gut-brain interaction. It also describes the animal models that are currently being used to gain a better understanding of the pathogenesis of IBS. Finally, it discusses ideas relating IBS in the future, including a brief glimpse at acupuncture as a treatment modality.

We present the following article in accordance with the Narrative Review reporting checklist (available at https:// dx.doi.org/10.21037/atm-21-2779).

\section{Methods}

\section{Pathophysiology of IBS}

\section{General pathophysiological features}

IBS has traditionally been described as a disorder of visceral hypersensitivity and GI motor disturbances, which lead to abdominal pain or discomfort and diarrhea or constipation, respectively $(5,8)$. However, the pathophysiology of IBS is not fully comprehended. Pathogenic factors, such as genetic susceptibility, food intolerance, gut-brain axis dysfunction, or innate immunity and dysbiosis issues, possibly contribute to this disorder. At present, it is still unknown which of these pathogenic factors can elicit or intensify IBS, largely because the symptoms show great interindividual differences (13). Therefore, for IBS, treatment often targets the patient's primary or most troublesome symptom, as opposed to being based on the underlying pathophysiology as with other organic GI diseases (14). Below we describe the main pathophysiological factors that are currently under investigation.

\section{Genetic factors}

Numerous lines of evidence show that the genetic risk for IBS varies between complex polygenic individuals who have combinations of common variants and those with atypical single gene aberrations $(15,16)$. For instance, a mutation in SC5NA, which is a sodium ion channel gene, was determined to be linked to abdominal pain in patients with IBS (17). Furthermore, several single nucleotide polymorphism (SNP) studies have detected polymorphisms in IBS pathogenesis-related genes, including those related to serotonin signaling $(18,19)$, immune regulation, epithelial barrier function (20), bile acid synthesis (21), and cannabinoid receptors (22). A genome-wide association study (GWAS) also revealed that KDELR2, a KDEL endoplasmic reticulum protein retention receptor, and GRID2IP, a glutamate receptor-interacting protein, were connected with the risk of developing IBS (23). However, because these studies had small cohorts and produced no evidence of prominent structural abnormalities, the role of genetics in IBS development has generally remained unclear. 
More than 60 candidate genes have been investigated in relation to IBS, but the data on only one gene, the TNFSF15 gene, has been convincing. An association of this gene with IBS was unearthed in an early study (24), and this finding was verified in independent cohorts $(20,25,26)$. Altogether, the association of TNFSF15 with IBS is worth further investigation, especially as this gene has involvement in the intestinal inflammatory response (27).

More recently, epigenetic factors, such as DNA methylation, have been linked to IBS. For example, one study used genome-wide methylation scanning to uncover differential DNA methylation in several CpG sites in the peripheral blood mononuclear cells (PBMCs) of patients with IBS in comparison to healthy controls (28). In addition to DNA methylation changes in PBMCs, epigenetic changes associated with IBS were also identified in the colonic mucosa. Increases in methylation have also been identified in stress-related genes, including NR3C1, CRHR1, and brain-derived neurotrophic factor (BDNF) (29). These findings show that DNA methylation changes are important pathophysiologic mechanisms in IBS that are worthy of further investigation.

\section{The role of the gut microbiota}

The pathogenesis of IBS is also associated with changes in the gut microbiota, which alter the immunity and integrity of the gut and further modulate the gut-brain axis and the gut neuromuscular junction (30). Under normal circumstances, the mucosal epithelium, which is where the stimulation of homeostatic immune responses occurs, upholds the integrity of the barrier and maintains tolerance to commensal bacteria by restricting microbes to the surface or the intestinal lumen. This process permits bacteria to steadfastly colonize the intestine and undergo cooperative functions. However, when this barrier is broken by invading inflammatory agents, pathogens, or other factors which aggravate the immune response, severe inflammation occurs. This inflammatory reaction affects the intestinal milieu, because it alters the composition of the gut microbiota (31). A number of studies have reported the diversity of the gut microbiome and how it is related to the severity of IBS (32-34), However, comparisons of the abundance of the microbiota in patients with the different IBS-subtypes have scarcely been undertaken. Thus, exploration of the gut microbiome is important in further understanding the pathophysiology of IBS.

To extend upon the importance of the gut microbiome, there is growing evidence to suggest that maintaining the appropriate diversity of gut microbiota is not only essential for gut health but is also critical for the normal physiological function of other organs, especially the brain. In neonates and older people, the occurrence of microbial imbalance, termed dysbiosis, has the potential to profoundly affect brain function. This is because the brain depends upon the metabolic products of gut microbes $(35,36)$. Thus, we will next focus in more detail on gut-brain interactions, with a particular focus on the autonomic nervous system (ANS), central nervous system (CNS), stress system, and corticotropin-releasing factor (CRF) system, as well as intestinal response.

\section{Gut-brain interactions}

\section{Defining the gut-brain axis}

The gut-brain axis (Figure 1) comprises the enteric nervous system (ENS), the CNS, the gut wall in the periphery, and the hypothalamo-pituitary-adrenal (HPA) axis (37). The communication between the gut and the CNS is bidirectional and is centered upon the neural, endocrine, and neuroimmune pathways (38). At the physiological level, the GI tract sends signals that affect the brain, resulting in alterations in immune function, secretion, and motility (36). Thus, this axis serves as a key communication hub in the regulation of food intake, digestion, and the sensation of adequate control of gut and bowel movements. Structural and functional disruptions of the gut-brain axis alter the nervous system's reflexive and perceptual responses, which can potentially instigate GI disorders, such as IBS (39).

\section{Gut-brain interaction: crosstalk between the CNS and ANS}

The vagus nerve encompasses thousands of nerve endings, among which $80 \%$ are afferent, carrying stimuli toward the $\mathrm{CNS}$ and brain. The vagus nerve is the main communication conduit between the brain and the microbiota (Figure 1). The pathways involved in this communication are also responsible for transmitting efferent signals from the CNS to the intestinal wall (40). The vagus nerve is an important component of the ANS, and the sympathoadrenomedullar axis is the specific component of the ANS that participates in acute stress response. The sympathoadrenal and HPA axes are considered to be the key constituents of the stress response system in vertebrates (41). Multiple anxiety-related psychiatric disorders and stress-sensitive pain syndromes arise due to alterations of this multifaceted system (42).

Correcting the crosstalk between the ANS and CNS 


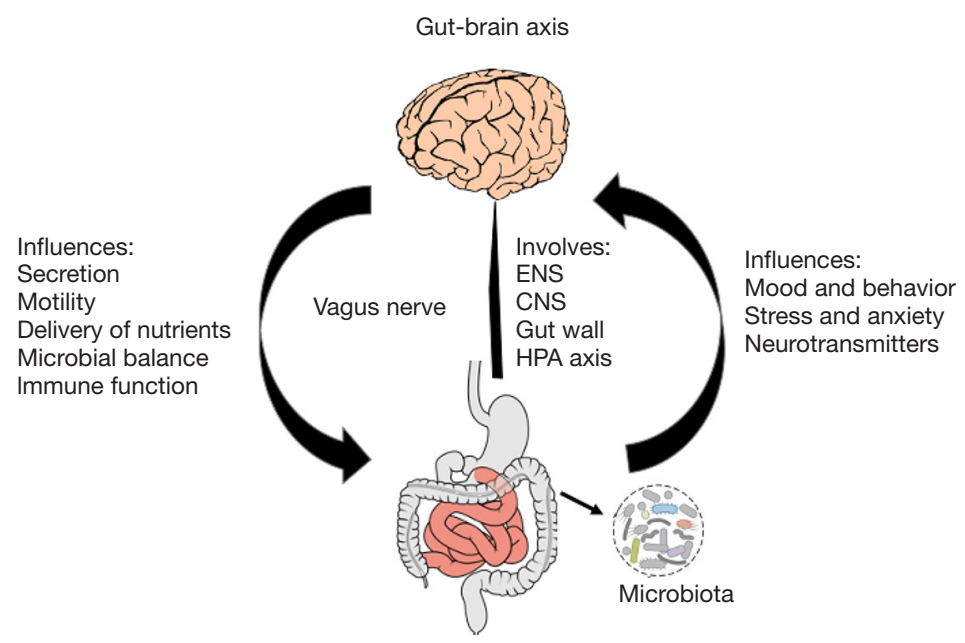

Figure 1 The gut-brain axis. The vagus nerve provides a link between the gut and the brain. This connection involves the ENS, the CNS, the gut wall at the periphery, and the HPA axis. Alterations in the gut microbiota can influence mood, behavior, stress, anxiety, and neurotransmitters. Imbalance of the gut microbiota affects the signals sent by the gut to the brain, resulting in alterations in secretion, motility, nutrient delivery, microbial balance, and immune function. Together, these disruptions contribute to IBS symptoms. ENS, enteric nervous system; CNS, central nervous system; HPA, hypothalamo-pituitary-adrenal; IBS, irritable bowel syndrome.

in an important function in IBS prevention. Disruption of the ANS at any level, including in the form of decreased parasympathetic and increased sympathetic activity, can cause patients with IBS to experience distorted autonomic reflexes, accounting for a degree of awareness when it comes to extraintestinal symptoms and GI stimuli (43). For example, in one study, the relationship between restingstate brain function and in vitro measures of gut barrier function was examined in healthy women versus women with moderate to severe IBS. Specifically, the connections between transcellular permeability (evaluated by live bacterial passage of Salmonella typhimurium) and paracellular permeability and resting-state functional connectivity in the default mode network brain region were examined. Ultimately, the women with moderate to severe IBS and the healthy women exhibited statistical differences in terms of paracellular and transcellular epithelial permeability, and structural and functional brain features. Furthermore, among the women with IBS, those with lower epithelial permeability reported more severe IBS symptoms, which was associated with increased functional and structural connectivity in endogenous pain facilitation regions. Taken together, the results of the study revealed that the relationship between gut permeability and the brain is significantly altered in patients with IBS, and these findings may help to differentiate IBS subtypes (44).

\section{Gut-brain interaction: the role of CRF}

CRF is an endogenous 41 -amino acid neuropeptide that is released by endocrine cells in the paraventricular nuclei of the hypothalamus. CRF is a principal activator of the HPA axis (Figure 2) (45). It functions both centrally and peripherally to modulate how the body responds to stress and stimulates IBS symptoms (46). Understanding how $\mathrm{CRF}$ and its receptors (CRFR1 and CRFR2) affect immunity and motility via the gut-brain axis might unearth more clues regarding the pathophysiology of IBS (47). Animal models can aid in this process. For instance, in one study, the administration of CRF to low-anxiety rats induced colonic hypersensitivity, which was blocked by pretreatment with a CRFR1 antagonist (48). Further, another study reported that fecal pellet output by rats under water-avoidance stress (WAS) increased following CRF administration; however, CRF receptor antagonists blocked this effect (49). Ultimately, the binding of CRF to its receptors induces changes in smooth muscle contractility, mucosal transport, mucosal permeability, and visceral pain sensitivity, all of which are correlated with the colonic manifestations of IBS (50).

Patients with IBS have been reported to show CRF-mediated colonic hypermotility and HPA axis hyperresponsiveness (51), and it is possible that sex plays a role in these differences (52). One study examined the HPA axis, colonic motility, and autonomic reactions following 


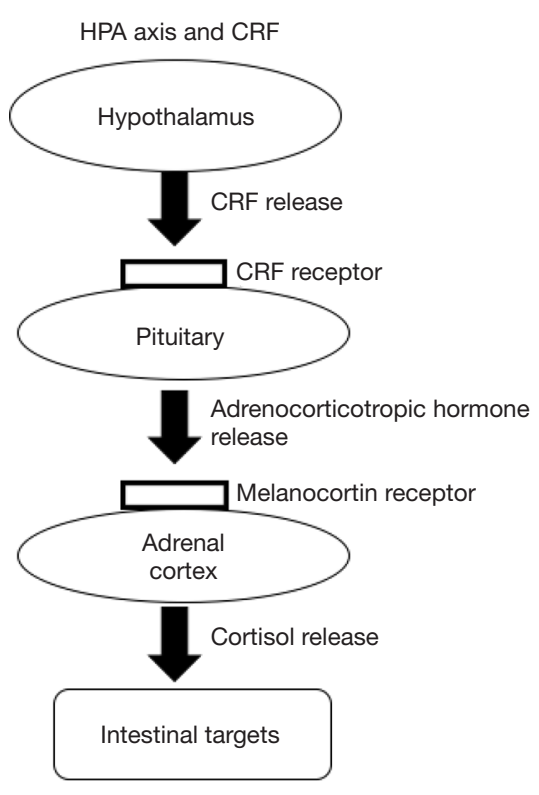

Figure 2 The HPA axis and CRF. CRF is the main activator of the HPA axis and is released by the hypothalamus in the brain. The receptor for CRF is found on the pituitary gland. Upon ligation, the pituitary releases adrenocorticotropic hormone, and its receptor is found in the adrenal cortex. By this point, the HPA axis is fully activated and this triggering of the adrenal cortex stimulates the release of cortisol, which in turn stimulates intestinal cells, causing some of the symptoms associated with IBS. HPA, hypothalamo-pituitary-adrenal; CRF, corticotropin-releasing factor; IBS, irritable bowel syndrome.

CRF administration, and also examined brain activity alterations in patients with IBS. After CRF administration, the male IBS group showed a greater increase in colonic motility than the male controls; in contrast, in comparison to their controls, the female IBS group displayed changes in sympathovagal balance and had a reduced basal parasympathetic tone. The study suggested that treatments that act centrally might aid in reducing the stress-induced physical symptoms of IBS (53). Ultimately, more studies into the mechanistic network of the central CRF system and the GI CRF system at a local level will improve our knowledge of the gut-brain axis.

\section{Gut-brain interaction: stress}

Psychological stress is recognized as a trigger of IBS and may have a critical influence on the gut-brain axis (54). Stress is known to affect intestinal motility and permeability, visceral sensitivity, immune responses, and gut microbiota composition (55). The underlying mechanism of the role of stress in IBS is believed to be related to the secretion of proinflammatory cytokines, which activates the HPA and hypothalamic-ANS axes, inducing the release of CRF, adrenocorticotropic hormone, and cortisol, which subsequently affects gut homeostasis (56). The connection between stress and IBS is evident, and due to the link with the gut-brain axis, the management of stress and stress-induced responses is a critical aspect of therapeutic intervention for patients with IBS.

\section{Animal models used to study the gut-brain interaction in IBS}

\section{Overview of animal models}

A variety of animal models have been developed to study the pathophysiology of IBS. These models involve the use of chemical and/or mechanical means to stimulate physiological or psychological stress, such as drugs, restraint stress, WAS, maternal separation (MS), cold-water stomach irrigation, or electric foot shock (57-61). Although none of these current models are considered ideal, we can still learn a great deal about the gut-brain axis and its role in IBS through examining them. Below, we review three of the most frequently used models.

\section{The wrap restraint stress (WRS) model}

The WRS model was first utilized more than 30 years ago and was initially believed to be a good method for modelling human IBS (62). However, as the years passed, deeper investigations into this model showed this consideration to not be entirely true, and presently, no animal model that accurately mimics IBS exists. The WRS model includes the forced immobilization of the animal once for at least 2 hours. The efficacy of this procedure is validated by the animal immediately developing hyperalgesia (63), small intestinal transit inhibition, stimulation of large intestinal activity, and increased fecal excretion (62). WRS-induced rats show low-grade mucosal inflammation that occurs with significant increases in mast cells and eosinophylic granulocytes (64), which is consistent with what is observed in human IBS colonic biopsies (65). The literature supports the suitability of the WRS model for partial replication of the principal symptoms observed in IBS, namely dysmotility and hypersensitivity. However, whether the WRS model is suitable for investigating potential drugs to treat IBS remains to be determined (66). Furthermore, it is worth noting that the WRS model is not able to show the chronic 
course of IBS (67). In fact, when the restraint is applied repetitively in an effort to enhance its acute effect, adaptive responses are activated (66), and this is the opposite of what occurs in human patients with IBS.

\section{Chronic stressor models}

Both childhood trauma of various causes and recurrent stress conditions in adulthood share significant associations with IBS (68). Thus, pre-existing or repetitively occurring adverse conditions are associated with the manifestation and continuation of IBS symptoms (69). Based on these clinical observations, researchers have developed animal models that mimic these chronic stressors. There are two main models of chronic stress, including the MS model, which mimics childhood trauma, and the WAS model, which mimics repetitive stress conditions in adults.

The most common MS model involves separating pups from their mother for 3 hours a day during the first 2 weeks of life. Maternal care affects the HPA axis, as well as cognitive and emotional functions (70). The purpose of the MS animal model is to induce stable alterations in the CNS (71). The model also alters large intestinal function, thus promoting the induction of visceral hypersensitivity to colorectal distension in the adult animals (72) and colonic mast cell hyperplasia (65), which are two characteristic features of IBS.

An interesting feature of the MS model is that female animals show greater sensitivity than males $(66,72)$. Remarkably, the occurrence of IBS is two-fold greater in women than in men, which is independent of the activating factors (69). Therefore, the MS model holds validity in terms of its consistency with the hypothetical pathogenesis of IBS (67).

Daily chronic stress has been shown to predict the severity of IBS symptoms (73). The WAS model is considered to be one of the most efficient psychological stressors fitting these criteria (74). WAS is known to increase fecal pellet output, colonic mast cell count, mucosal cytokine levels, and intestinal permeability (75). For example, colonic movement, mucosal mast cell count, cytokine levels, and visceromotor response to colorectal distension were all assessed in male and female rats following repeated exposure to WAS. In rats of both sexes, the repeated WAS group produced significantly more fecal pellets than did the non-stressed group; however, the output rate was increased in females compared to males. Furthermore, the colonic mucosal cytokine levels were higher in the WAS group than in the non-stressed group, but only among females (76).
These data demonstrate that the WAS model is useful for examining the colonic disruptions that occur in clinical IBS.

IBS is a complex disorder, and despite these IBS experimental models having limitations, they have allowed researchers to verify psychosocial stressors as important contributors to the cause and time-course of gut symptomatology, therefore making analyses of the gut-brain axis feasible. Moreover, the three models described above stimulate visceral hypersensitivity, which is one of the key indicators of IBS. Overall, the utilization of animal models in the study of IBS has been proven to be valuable.

\section{Discussion}

\section{Investigating the mechanism of gut-brain interaction}

\section{Proteomic changes in IBS}

Part of the challenge researchers face in understanding the pathophysiology of IBS results from its heterogeneity. In clinical biology, the use of mass spectrometry offers a means to interrogate the proteome of biological samples, such as blood, urine, saliva, or tissue, for the purpose of biomarker discovery (77). For instance, one study examined the urine proteome of women with IBS and subclassified each case on the basis of the predominant symptoms. Mass spectrometry analysis revealed differences in the urine proteome levels depending on the subclass. Among the proteins displaying distinctive changes were those known to be related to intestinal function homeostasis and inflammatory response, which indicates that future studies should further investigate and validate urinary protein markers in female patients with IBS, as they might serve as a diagnostic tool (78).

In addition to biological samples, animal models of IBS have also been used to uncover proteomic changes. For instance, a rat IBS model was established using a combination of MS and WAS. The colons of the rats and their respective controls were subjected to mass spectrometry analysis. A number of the upregulated proteins in the IBS rats were related to stress-mediated modulation of GI motility and/or mucosal inflammation (79). This finding further contributes to the understanding of the gut-brain interaction in IBS; however, because only the colon was examined, the involvement of the brain proteome could not be clearly defined. Subsequently, another group of researchers constructed a proteomics map for brain and colon tissue from rats, with the goal of identifying differentially expressed proteins between the IBS and control rats. In this study, the IBS rats were subjected to 
MS, WRS, or a combination of the two, and brain and gut tissues from these rats and their respective controls were assessed by mass spectrometry. Comprehensive and quantitative proteomic analysis of the brain and colon tissue in the IBS models demonstrated significant differences. The identification of these proteins contributes to our understanding and provides new evidence of the abnormal brain-gut interaction that occurs in IBS (80). The application of proteomics has enormous potential and will likely provide significant insight into improving the understanding and management of IBS.

\section{Pharmacogenetics and gut-brain interaction in IBS}

Managing IBS remains challenging, and current pharmacotherapeutic approaches usually follow a trialand-error process that results in modest or no symptomatic improvement (81). In an effort to improve upon this strategy, more studies are using pharmacogenetics, which covers the effect of genetic variability on patients' responses to medications (82). Applying the concepts of pharmacogenetics to the management algorithm of IBS may help to improve the outcome of specific therapies by maximizing efficacy and minimizing toxicity.

Pharmacogenetics modulates responses to therapy in IBS via pharmacokinetics (the modulation of drug metabolism) and pharmacodynamics (the changes affecting receptors or transporters that are involved in drugs' mechanisms of action) (82). With regard to pharmacokinetics, cytochrome P-450 (CYP450) enzymes, such as CYP2C19 and CYP2D6, play a role in the metabolism of many medications used to treat IBS, specifically antidepressants. Possessing more than 100 genetic variants, the CYP2D6 enzyme is highly polymorphic, and the number of functional CYP2D genes is correlated with the metabolism of the antidepressant nortriptyline (83). However, how the metabolism of antidepressants affects the therapeutic response has not been studied extensively in patients with DGBIs. Therefore, future studies should focus on the pharmacogenetics of drug metabolism and tailor the choice and dose of antidepressant and for each patient based on their genetic makeup. This approach may help to minimize toxicities and maximize benefits.

Regarding the pharmacodynamic aspect of pharmacogenetics, studies to date have focused on serotonin receptors as therapeutic targets in IBS. Serotonin $(5-H T)$ is an essential neurotransmitter and paracrine signaling molecule in the gut and has been implicated in a variety of diverse physiologic functions (84). Thus, targeting serotonin receptors has been a research focus in IBS over other pathways. For instance, 5-HT3 receptor antagonists have been used. However, the 5-HT3 receptor genes are polymorphic, and these variants are thought to affect the treatment response (85). The roles of the different 5 -HT3 receptor variants are now detailed in an electronic database (85), which allows genetic and pharmacogenetic data to be continuously gathered as a reference for potential clinical use. With this resource, studies can now be designed to assess polymorphisms in 5 -HT3 receptor genes, which will contribute to the management of DGBIs. Ultimately, 5-HT3 receptor gene polymorphisms should be monitored before IBS treatment, especially when the use of 5-HT3 receptor antagonists is a possibility.

Tryptophan hydroxylase 1 (THP1), which is the ratelimiting enzyme involved in the synthesis of 5-HT, is also known to possess polymorphisms. The frequency of certain THP1 genotypes is significantly higher among patients with IBS who respond to the 5-HT3R antagonist ramosetron than among non-responders (86). This finding may assist in the identification of subgroups of patients with IBS who have a greater chance of responding to new agents, such as ramosetron. Furthermore, studies examining THP1 genetic polymorphisms will further aid in elucidating the role of serotonin as a biomarker for IBS.

\section{Future directions}

It is evident that the pathology of IBS is complicated and includes fluctuations in the microbiota, immunological changes, and regulation of the gut-brain signaling axis; owing to this, success with therapeutic approaches has largely been limited. However, there are endless possibilities for future research on IBS, and advances in technology have enabled the development of novel methods for examining this disease.

The revolution and development of human intestinal microfluidic organ-on-a-chip models have transformed the approach to examining intestinal physiology and pathophysiology (87). The co-culture of intestinal microbes with viable epithelium for more than 1 day is generally challenging with conventional culture models, even using intestinal organoid cultures. Additionally, traditional cell culture methods are not capable of replicating the physiological environment of the intestines, such as the formation of distinctive intestinal villi and mucus production, and the main intestinal differentiated functions, such as CYP450-based drug metabolism. 
Furthermore, the rapid overgrowth of commensal bacteria contaminates human cell cultures. However, a two-channel microfluidic organ chip device lined with human $\mathrm{CaCO}_{2}$ intestinal epithelial cells cultured under dynamic fluid flow and peristalsis-like mechanical deformations has been developed. This device allows for the stable co-culture of a mucus-producing human villus intestinal epithelium with up to eight different human commensal gut microorganism strains. These co-cultures can be maintained for weeks under aerobic conditions $(88,89)$. This technology represents a huge improvement over traditional culture systems.

However, the human gut microbiome comprises hundreds of different types of bacteria, a number of which will not grow in this environment, because they are obligate anaerobes. Therefore, another version of this organ-on-achip model was needed. Employing a microfluidic intestineon-a-chip system, Jalili-Firoozinezhad et al. co-cultured live human intestinal epithelium with stable communities of aerobic and anaerobic human gut microbiota, which permitted the control and real-time assessment of physiologically relevant oxygen gradients (90). The establishment of this intestine-on-a-chip has the potential to aid in the development of microbiome-related therapeutics, nutraceuticals, and probiotics.

Another area of IBS research that holds potential in the future is metabolomics. Two main technologies can be utilized in metabolomics studies: mass spectrometry and nuclear magnetic resonance spectroscopy (91). Comparative metabolomic studies using the microbiota from unhealthy and healthy patients have identified unique metabolites that have the potential to serve as diagnostic or prognostic biomarkers. A number of metabolites can be altered by food intake, and these alterations can be used to identify perturbations in metabolic pathways in order to distinguish a normal/healthy gut from a dysfunctional/unhealthy one. The exploration of the gut microbiota and the relationships between the microbial metabolites and the host may provide important clues on the pathophysiology of IBS (91). In the future, researchers and clinicians should be encouraged to not exclusively rely on symptoms for diagnosis and results; instead, they should obtain a better understanding of the variabilities in the concentrations of the microbial-derived metabolites that contribute to the symptoms and severity of IBS (92). A better understanding of IBS metabolomics may reveal more about its pathophysiology.

As research continues, treatments will also evolve. One strategy that holds promise is acupuncture, which is reported to be a favorable alternative for the treatment of functional GI disorders (93). Single-center studies suggest that acupuncture has greater efficacy for patients with IBS than pinaverium bromide and lactulose. Importantly, these studies observed greater symptomatic improvement, better long-term effects, lower relapse rates, and fewer side effects in patients treated with acupuncture $(94,95)$. However, as they were single-center studies with small patient samples, a multicenter randomized controlled trial of high methodologic quality was conducted to verify their findings. This large study of 531 patients compared acupuncture to PEG 4000/pinaverium bromide, and found that acupuncture alleviated IBS symptoms, as assessed using the IBS severity scoring system (96), and improved patients' quality-oflife over the 6-week treatment period and the 12 -week follow-up period. Importantly, the patients did not experience any adverse effects (97). Therefore, acupuncture appears to provide great benefits and might be a potential treatment for IBS in the future. However, more studies focusing on patient expectations and the placebo effect, and assessing the various IBS subtypes are necessary. In addition, the underlying mechanism of acupuncture in the treatment of IBS remain unclear. However, there is evidence to support that neurotransmitters are involved. Serotonin reuptake transporter (SERT) gene polymorphisms are implicated in the disturbances of GI functions in IBS. The SERT gene is responsible for controlling the synaptic concentration of 5-HT. Based on a study that looked at the safety of acupuncture for IBS while investigating the role of genetics in IBS, the effect of acupuncture on IBS symptoms may also be associated with the patients' polymorphisms in the SERT-related genes (98).

Because our knowledge of the pathophysiology of IBS is still lacking, strategies designed to treat the disease focus on symptom management rather than disease modification. These strategies include lifestyle and diet changes, psychological treatments, and the use of pharmacological agents (99). However, patients with IBS find these treatments unsatisfactory, and the adverse events associated with pharmacologic agents can result in treatment discontinuation. Therefore, there is a great need for alternative therapies to be discovered. The idea of manipulating the microbiota to treat IBS is compelling (100). A variety of meta-analyses reveal that probiotics, in general, benefit patients with IBS (101). However, a major problem for the field of IBS research is that these studies are of poor quality and include small populations, variable end points, and different organisms. Thus, the findings are difficult to interpret and compare. 
In addition, most probiotics studies in IBS patients do not compare the IBS subtypes (101). Therefore, there is a need to create better studies to assess the effect of probiotics in IBS patients.

\section{Conclusions}

This review has summarized the pathophysiology of IBS, with a specific focus on gut-brain interaction. There is clear evidence that gut-brain interaction is an important aspect for the development of future treatments and diagnosis of IBS. We have also described the various animal models that are used to demonstrate the effect that stress has on GI motility, epithelial secretion, microbiota, inflammatory response, abdominal muscle contraction, and pain response, and have outlined the utility of these animal models. Furthermore, CRF and its receptors are important, because stress activates the CRF system, which in turn stimulates cells in the GI tract, leading to IBS phenotypes, including increased permeability, mucin secretion, ion secretion, visceral hypersensitivity, and proinflammatory cytokine release. Overall, the current evidence shows that there is a clear demand for studies that uncover the mechanism of the central CRF and local GI CRF systems, in order to postulate new visions for understanding the gut-brain axis. Furthermore, technologies, including organ-on-a-chip models and metabolomics, provide novel means of digging deeper into the pathology of IBS, which will be crucial for the development of treatments, such as acupuncture, in the future. Finally, we have provided a brief glimpse into acupuncture as a potentially important treatment modality for IBS which can alleviate patients' symptoms and improve their quality of life, suggesting that it will be a key focus in future studies.

\section{Acknowledgments}

Funding: This study was supported by the National Natural Science Foundation of China (grant Nos. 81973936, 81704166, and 81874457).

\section{Footnote}

Reporting Checklist: The authors have completed the Narrative Review reporting checklist. Available at https:// dx.doi.org/10.21037/atm-21-2779

Conflicts of Interest: All authors have completed the ICMJE uniform disclosure form (available at https://dx.doi. org/10.21037/atm-21-2779). Dr. GMS reported that this work was funded by National Natural Science Foundation of China (grant Nos. 81973936, 81704166, and 81874457). The other authors have no conflicts of interest to declare.

Ethical Statement: The authors are accountable for all aspects of the work in ensuring that questions related to the accuracy or integrity of any part of the work are appropriately investigated and resolved.

Open Access Statement: This is an Open Access article distributed in accordance with the Creative Commons Attribution-NonCommercial-NoDerivs 4.0 International License (CC BY-NC-ND 4.0), which permits the noncommercial replication and distribution of the article with the strict proviso that no changes or edits are made and the original work is properly cited (including links to both the formal publication through the relevant DOI and the license). See: https://creativecommons.org/licenses/by-nc-nd/4.0/.

\section{References}

1. Drossman DA. Functional gastrointestinal disorders: what's new for Rome IV? Lancet Gastroenterol Hepatol 2016;1:6-8.

2. Occhipinti K, Smith JW. Irritable bowel syndrome: a review and update. Clin Colon Rectal Surg 2012;25:46-52.

3. Soares RL. Irritable bowel syndrome: a clinical review. World J Gastroenterol 2014;20:12144-60.

4. Sperber AD, Drossman DA. Irritable bowel syndrome: a multidimensional disorder cannot be understood or treated from a unidimensional perspective. Therap Adv Gastroenterol 2012;5:387-93.

5. Talley NJ, Spiller R. Irritable bowel syndrome: a little understood organic bowel disease? Lancet 2002;360:555-64.

6. Mearin F, Lacy BE, Chang L, et al. Bowel disorders. Gastroenterology 2016. [Epub ahead of print]. doi: 10.1053/j.gastro.2016.02.031.

7. Boyce PM, Talley NJ, Burke C, et al. Epidemiology of the functional gastrointestinal disorders diagnosed according to Rome II criteria: an Australian population-based study. Intern Med J 2006;36:28-36.

8. Drossman DA. The functional gastrointestinal disorders and the Rome III process. Gastroenterology 2006;130:1377-90.

9. Sperber AD, Bangdiwala SI, Drossman DA, et al. 
Worldwide prevalence and burden of functional gastrointestinal disorders, results of Rome foundation global study. Gastroenterology 2021;160:99-114.e3.

10. Houghton LA, Heitkemper M, Crowell M, et al. Age, gender and women's health and the patient. Gastroenterology 2016. [Epub ahead of print]. doi: 10.1053/j.gastro.2016.02.017.

11. Lovell RM, Ford AC. Effect of gender on prevalence of irritable bowel syndrome in the community: systematic review and meta-analysis. Am J Gastroenterol 2012;107:991-1000.

12. Gwee KA. Irritable bowel syndrome in developing countries--a disorder of civilization or colonization? Neurogastroenterol Motil 2005;17:317-24.

13. Bellini M, Gambaccini D, Stasi C, et al. Irritable bowel syndrome: a disease still searching for pathogenesis, diagnosis and therapy. World J Gastroenterol 2014;20:8807-20.

14. Talley NJ, Holtmann G, Walker MM. Therapeutic strategies for functional dyspepsia and irritable bowel syndrome based on pathophysiology. J Gastroenterol 2015;50:601-13.

15. D'Amato M. Genes and functional GI disorders: from casual to causal relationship. Neurogastroenterol Motil 2013;25:638-49.

16. Gazouli M, Wouters MM, Kapur-Pojskić L, et al. Lessons learned--resolving the enigma of genetic factors in IBS. Nat Rev Gastroenterol Hepatol 2016;13:77-87.

17. Beyder A, Mazzone A, Strege PR, et al. Loss-offunction of the voltage-gated sodium channel NaV1.5 (channelopathies) in patients with irritable bowel syndrome. Gastroenterology 2014;146:1659-68.

18. Grasberger H, Chang L, Shih W, et al. Identification of a functional TPH1 polymorphism associated with irritable bowel syndrome bowel habit subtypes. Am J Gastroenterol 2013;108:1766-74.

19. Jun S, Kohen R, Cain KC, et al. Associations of tryptophan hydroxylase gene polymorphisms with irritable bowel syndrome. Neurogastroenterol Motil 2011;23:233-9, e116.

20. Wouters MM, Lambrechts D, Knapp M, et al. Genetic variants in CDC42 and NXPH1 as susceptibility factors for constipation and diarrhoea predominant irritable bowel syndrome. Gut 2014;63:1103-11.

21. Wong BS, Camilleri M, Carlson P, et al. Increased bile acid biosynthesis is associated with irritable bowel syndrome with diarrhea. Clin Gastroenterol Hepatol 2012;10:1009-15.e3.

22. Camilleri M, Kolar GJ, Vazquez-Roque MI, et al.
Cannabinoid receptor 1 gene and irritable bowel syndrome: phenotype and quantitative traits. Am J Physiol Gastrointest Liver Physiol 2013;304:G553-60.

23. Ek WE, Reznichenko A, Ripke S, et al. Exploring the genetics of irritable bowel syndrome: a GWA study in the general population and replication in multinational casecontrol cohorts. Gut 2015;64:1774-82.

24. Zucchelli M, Camilleri M, Andreasson AN, et al. Association of TNFSF15 polymorphism with irritable bowel syndrome. Gut 2011;60:1671-7.

25. Czogalla B, Schmitteckert S, Houghton LA, et al. A metaanalysis of immunogenetic Case-Control Association Studies in irritable bowel syndrome. Neurogastroenterol Motil 2015;27:717-27.

26. Swan C, Duroudier NP, Campbell E, et al. Identifying and testing candidate genetic polymorphisms in the irritable bowel syndrome (IBS): association with TNFSF15 and TNF $\alpha$. Gut 2013;62:985-94.

27. Kadiyska T, Tourtourikov I, Popmihaylova AM, et al. Role of TNFSF15 in the intestinal inflammatory response. World J Gastrointest Pathophysiol 2018;9:73-8.

28. Mahurkar S, Polytarchou C, Iliopoulos D, et al. Genome-wide DNA methylation profiling of peripheral blood mononuclear cells in irritable bowel syndrome. Neurogastroenterol Motil 2016;28:410-22.

29. Mahurkar-Joshi S, Videlock EJ, Iliopoulos D, et al. 1090-epigenetic changes in blood cells and colonic mucosa are associated with irritable bowel syndrome (IBS). Gastroenterology 2018;154:S-214.

30. Carroll IM, Ringel-Kulka T, Keku TO, et al. Molecular analysis of the luminal- and mucosal-associated intestinal microbiota in diarrhea-predominant irritable bowel syndrome. Am J Physiol Gastrointest Liver Physiol 2011;301:G799-807.

31. Pédron T, Sansonetti P. Commensals, bacterial pathogens and intestinal inflammation: an intriguing ménage à trois. Cell Host Microbe 2008;3:344-7.

32. Keselman A, Li E, Maloney J, et al. The microbiota contributes to CD8+ t cell activation and nutrient malabsorption following intestinal infection with giardia duodenalis. Infect Immun 2016;84:2853-60.

33. Reti KL, Tymensen LD, Davis SP, et al. Campylobacter jejuni increases flagellar expression and adhesion of noninvasive Escherichia coli: effects on enterocytic Tolllike receptor 4 and CXCL-8 expression. Infect Immun 2015;83:4571-81.

34. Rigsbee L, Agans R, Shankar V, et al. Quantitative profiling of gut microbiota of children with diarrhea- 
predominant irritable bowel syndrome. Am J Gastroenterol 2012;107:1740-51.

35. Dinan TG, Stilling RM, Stanton C, et al. Collective unconscious: how gut microbes shape human behavior. J Psychiatr Res 2015;63:1-9.

36. Mayer EA, Knight R, Mazmanian SK, et al. Gut microbes and the brain: paradigm shift in neuroscience. J Neurosci 2014;34:15490-6.

37. Collins SM, Bercik P. The relationship between intestinal microbiota and the central nervous system in normal gastrointestinal function and disease. Gastroenterology 2009; 136:2003-14.

38. Mulak A, Bonaz B. Irritable bowel syndrome: a model of the brain-gut interactions. Med Sci Monit 2004; 10:RA55-62.

39. Clarke G, Quigley EM, Cryan JF, et al. Irritable bowel syndrome: towards biomarker identification. Trends Mol Med 2009; 15:478-89.

40. Carabotti M, Scirocco A, Maselli MA, et al. The gutbrain axis: interactions between enteric microbiota, central and enteric nervous systems. Ann Gastroenterol 2015;28:203-9.

41. Tanaka Y, Kanazawa M, Kano M, et al. Relationship between sympathoadrenal and pituitary-adrenal response during colorectal distention in the presence of corticotropin-releasing hormone in patients with irritable bowel syndrome and healthy controls. PLoS One 2018;13:e0199698.

42. Fichna J, Storr MA. Brain-gut interactions in IBS. Front Pharmacol 2012;3:127.

43. Azpiroz F. Gastrointestinal perception: pathophysiological implications. Neurogastroenterol Motil 2002;14:229-39.

44. Witt ST, Bednarska O, Keita ÅV, et al. Interactions between gut permeability and brain structure and function in health and irritable bowel syndrome. Neuroimage Clin 2019;21:101602.

45. Aguilera G, Subburaju S, Young S, et al. The parvocellular vasopressinergic system and responsiveness of the hypothalamic pituitary adrenal axis during chronic stress. Prog Brain Res 2008;170:29-39.

46. Taché Y, Kiank C, Stengel A. A role for corticotropinreleasing factor in functional gastrointestinal disorders. Curr Gastroenterol Rep 2009;11:270-7.

47. Chatoo M, Li Y, Ma Z, et al. Involvement of corticotropin-releasing factor and receptors in immune cells in irritable bowel syndrome. Front Endocrinol (Lausanne) 2018;9:21.

48. Greenwood-Van Meerveld B, Johnson AC, Cochrane S, et al. Corticotropin-releasing factor 1 receptor-mediated mechanisms inhibit colonic hypersensitivity in rats. Neurogastroenterol Motil 2005;17:415-22.

49. Maillot C, Million M, Wei JY, et al. Peripheral corticotropin-releasing factor and stress-stimulated colonic motor activity involve type 1 receptor in rats. Gastroenterology 2000;119:1569-79.

50. Chang L. The role of stress on physiologic responses and clinical symptoms in irritable bowel syndrome. Gastroenterology 2011;140:761-5.

51. Dinan TG, Quigley EM, Ahmed SM, et al. Hypothalamicpituitary-gut axis dysregulation in irritable bowel syndrome: plasma cytokines as a potential biomarker? Gastroenterology 2006;130:304-11.

52. Videlock EJ, Shih W, Adeyemo M, et al. The effect of sex and irritable bowel syndrome on HPA axis response and peripheral glucocorticoid receptor expression. Psychoneuroendocrinology 2016;69:67-76.

53. Kano M, Muratsubaki T, Van Oudenhove L, et al. Altered brain and gut responses to corticotropin-releasing hormone $(\mathrm{CRH})$ in patients with irritable bowel syndrome. Sci Rep 2017;7:12425.

54. Sgambato D, Miranda A, Ranaldo R, et al. the role of stress in inflammatory bowel diseases. Curr Pharm Des 2017;23:3997-4002.

55. Qin HY, Cheng CW, Tang XD, et al. Impact of psychological stress on irritable bowel syndrome. World J Gastroenterol 2014;20:14126-31.

56. Dieleman GC, Huizink AC, Tulen JH, et al. Alterations in HPA-axis and autonomic nervous system functioning in childhood anxiety disorders point to a chronic stress hypothesis. Psychoneuroendocrinology 2015;51:135-50.

57. Funatsu T, Takeuchi A, Hirata T, et al. Effect of ramosetron on conditioned emotional stress-induced colonic dysfunction as a model of irritable bowel syndrome in rats. Eur J Pharmacol 2007;573:190-5.

58. Murakami T, Kamada K, Mizushima K, et al. Changes in intestinal motility and gut microbiota composition in a rat stress model. Digestion 2017;95:55-60.

59. Qin HY, Xiao HT, Wu JC, et al. Key factors in developing the trinitrobenzene sulfonic acid-induced postinflammatory irritable bowel syndrome model in rats. World J Gastroenterol 2012;18:2481-92.

60. Winston JH, Xu GY, Sarna SK. Adrenergic stimulation mediates visceral hypersensitivity to colorectal distension following heterotypic chronic stress. Gastroenterology 2010;138:294-304.e3.

61. Zou N, Lv H, Li J, et al. Changes in brain G proteins 
and colonic sympathetic neural signaling in chronic-acute combined stress rat model of irritable bowel syndrome (IBS). Transl Res 2008;152:283-9.

62. Williams CL, Villar RG, Peterson JM, et al. Stressinduced changes in intestinal transit in the rat: a model for irritable bowel syndrome. Gastroenterology 1988;94:611-21.

63. Vannucchi MG, Evangelista S. Experimental models of irritable bowel syndrome and the role of the enteric neurotransmission. J Clin Med 2018;7:4.

64. Traini C, Evangelista S, Girod V, et al. Repeated otilonium bromide administration prevents neurotransmitter changes in colon of rats underwent to wrap restraint stress. J Cell Mol Med 2017;21:735-45.

65. Bashashati M, Moossavi S, Cremon C, et al. Colonic immune cells in irritable bowel syndrome: A systematic review and meta-analysis. Neurogastroenterol Motil 2018. doi: 10.1111/nmo.13192.

66. Larauche M, Mulak A, Taché Y. Stress and visceral pain: from animal models to clinical therapies. Exp Neurol 2012;233:49-67.

67. Mayer EA, Collins SM. Evolving pathophysiologic models of functional gastrointestinal disorders. Gastroenterology 2002;122:2032-48.

68. Mayer EA, Labus JS, Tillisch K, et al. Towards a systems view of IBS. Nat Rev Gastroenterol Hepatol 2015;12:592-605.

69. Moloney RD, O'Mahony SM, Dinan TG, et al. Stressinduced visceral pain: toward animal models of irritablebowel syndrome and associated comorbidities. Front Psychiatry 2015;6:15.

70. Fish EW, Shahrokh D, Bagot R, et al. Epigenetic programming of stress responses through variations in maternal care. Ann N Y Acad Sci 2004;1036:167-80.

71. Szyf M, Weaver I, Meaney M. Maternal care, the epigenome and phenotypic differences in behavior. Reprod Toxicol 2007;24:9-19.

72. Rosztóczy A, Fioramonti J, Jármay K, et al. Influence of sex and experimental protocol on the effect of maternal deprivation on rectal sensitivity to distension in the adult rat. Neurogastroenterol Motil 2003;15:679-86.

73. Elsenbruch S, Rosenberger C, Bingel U, et al. Patients with irritable bowel syndrome have altered emotional modulation of neural responses to visceral stimuli. Gastroenterology 2010;139:1310-9.

74. Bradesi S, Schwetz I, Ennes HS, et al. Repeated exposure to water avoidance stress in rats: a new model for sustained visceral hyperalgesia. Am J Physiol Gastrointest Liver
Physiol 2005;289:G42-53.

75. Larauche M, Mulak A, Taché Y. Stress-related alterations of visceral sensation: animal models for irritable bowel syndrome study. J Neurogastroenterol Motil 2011;17:213-34.

76. Lee JY, Kim N, Kim YS, et al. Repeated water avoidance stress alters mucosal mast cell counts, interleukin- $1 \beta$ levels with sex differences in the distal colon of Wister rats. J Neurogastroenterol Motil 2016;22:694-704.

77. Sun J, Chen F, Chen C, et al. Intestinal mRNA expression profile and bioinformatics analysis in a methamphetamineinduced mouse model of inflammatory bowel disease. Ann Transl Med 2020;8:1669.

78. Goo YA, Cain K, Jarrett M, et al. Urinary proteome analysis of irritable bowel syndrome (IBS) symptom subgroups. J Proteome Res 2012;11:5650-62.

79. Lopes LV, Marvin-Guy LF, Fuerholz A, et al. Maternal deprivation affects the neuromuscular protein profile of the rat colon in response to an acute stressor later in life. J Proteomics 2008;71:80-8.

80. Zhang B, Xue H, Wang W, et al. Comparative proteomic analysis of the brain and colon in three rat models of irritable bowel syndrome. Proteome Sci 2020;18:1.

81. Lucak S, Chang L, Halpert A, et al. Current and emergent pharmacologic treatments for irritable bowel syndrome with diarrhea: evidence-based treatment in practice. Therap Adv Gastroenterol 2017;10:253-75.

82. Halawi H, Camilleri M. Pharmacogenetics and the treatment of functional gastrointestinal disorders. Pharmacogenomics 2017;18:1085-94.

83. Camilleri $M$. The role of pharmacogenetics in nonmalignant gastrointestinal diseases. Nat Rev Gastroenterol Hepatol 2012;9:173-84.

84. Stasi C, Bellini M, Bassotti G, et al. Serotonin receptors and their role in the pathophysiology and therapy of irritable bowel syndrome. Tech Coloproctol 2014;18:613-21.

85. Celli J, Rappold G, Niesler B. The human serotonin type 3 receptor gene (HTR3A-E) allelic variant database. Hum Mutat 2017;38:137-47.

86. Shiotani A, Kusunoki H, Ishii M, et al. Pilot study of Biomarkers for predicting effectiveness of ramosetron in diarrhea-predominant irritable bowel syndrome: expression of S100A10 and polymorphisms of TPH1. Neurogastroenterol Motil 2015;27:82-91.

87. Bein A, Shin W, Jalili-Firoozinezhad S, et al. Microfluidic organ-on-a-chip models of human intestine. Cell Mol Gastroenterol Hepatol 2018;5:659-68.

88. Kim HJ, Li H, Collins JJ, et al. Contributions of 
microbiome and mechanical deformation to intestinal bacterial overgrowth and inflammation in a human guton-a-chip. Proc Natl Acad Sci U S A 2016;113:E7-15.

89. Park GS, Park MH, Shin W, et al. Emulating hostmicrobiome ecosystem of human gastrointestinal tract in vitro. Stem Cell Rev Rep 2017;13:321-34.

90. Jalili-Firoozinezhad S, Gazzaniga FS, Calamari EL, et al. A complex human gut microbiome cultured in an anaerobic intestine-on-a-chip. Nat Biomed Eng 2019;3:520-31.

91. Vernocchi P, Del Chierico F, Putignani L. Gut microbiota profiling: metabolomics based approach to unravel compounds affecting human health. Front Microbiol 2016;7:1144.

92. James SC, Fraser K, Young W, et al. Gut microbial metabolites and biochemical pathways involved in irritable bowel syndrome: effects of diet and nutrition on the microbiome. J Nutr 2020;150:1012-21.

93. Li H, He T, Xu Q, et al. Acupuncture and regulation of gastrointestinal function. World J Gastroenterol 2015;21:8304-13.

94. Li H, Pei LX, Zhou JL. Comparative observation on therapeutic effects between acupuncture and western medication for diarrhea-predominant irritable bowel syndrome. Zhongguo Zhen Jiu 2012;32:679-82.

95. Pei L, Zhu L, Sun J, et al. Constipation predominant irritable bowel syndrome treated with acupuncture

Cite this article as: Tang HY, Jiang AJ, Wang XY, Wang H, Guan YY, Li F, Shen GM. Uncovering the pathophysiology of irritable bowel syndrome by exploring the gut-brain axis: a narrative review. Ann Transl Med 2021;9(14):1187. doi: 10.21037/atm-21-2779 for regulating the mind and strengthening the spleen: a randomized controlled trial. Zhongguo Zhen Jiu 2015;35:1095-8.

96. Francis CY, Morris J, Whorwell PJ. The irritable bowel severity scoring system: a simple method of monitoring irritable bowel syndrome and its progress. Aliment Pharmacol Ther 1997;11:395-402.

97. Pei L, Geng H, Guo J, et al. Effect of acupuncture in patients with irritable bowel syndrome: a randomized controlled trial. Mayo Clin Proc 2020;95:1671-83.

98. Pei LX, Geng H, Chen H, et al. Acupuncture for irritable bowel syndrome: study protocol for a multicenter randomized controlled trial. Trials 2018;19:529.

99. Pietrzak A, Skrzydło-Radomańska B, Mulak A, et al. Guidelines on the management of irritable bowel syndrome: in memory of Professor Witold Bartnik. Prz Gastroenterol 2018;13:259-88.

100. Quigley EM, Flourie B. Probiotics and irritable bowel syndrome: a rationale for their use and an assessment of the evidence to date. Neurogastroenterol Motil 2007;19:166-72.

101. Shanahan F, Quigley EM. Manipulation of the microbiota for treatment of IBS and IBD-challenges and controversies. Gastroenterology 2014;146:1554-63.

(English Language Editor: J. Reynolds) 